\title{
Efficient Signal Processing Techniques for Exploiting Transmit Antenna Diversity on Fading Channels
}

\author{
Gregory W. Wornell, Member, IEEE, and Mitchell D. Trott, Member, IEEE
}

\begin{abstract}
A class of powerful and computationally efficient strategies for exploiting transmit antenna diversity on fading channels is developed. These strategies, which require simple linear processing at the transmitter and receiver, have attractive asymptotic characteristics. In particular, given a sufficient number of transmit antennas, these techniques effectively transform a nonselective Rayleigh fading channel into a nonfading, simple white marginally Gaussian noise channel with no intersymbol interference. These strategies, which we refer to as linear antenna precoding, can be efficiently combined with trellis coding and other popular error-correcting codes for bandwidth-constrained Gaussian channels. Linear antenna precoding requires no additional power or bandwidth and is attractive in terms of robustness and delay considerations. The resulting schemes have powerful and convenient interpretations in terms of transforming nonselective fading channels into frequency- and time-selective ones.
\end{abstract}

\section{INTRODUCTION}

$\mathbf{S}$ IGNAL processing has an increasingly important role to play in wireless communications systems for a host of applications. Examples include digital cellular networks and mobile radio, wireless LAN's and wireless local loops, digital audio and television broadcasting systems, and indoor wireless and personal communication systems. Indeed, accommodating the dramatic growth in demand for such services and meeting increasingly challenging performance specifications, will require that sophisticated signal processing algorithms be an integral part of next-generation systems.

In wireless applications, fading due to multipath propagation severely impacts system performance. However, the effects of fading can be substantially mitigated through the use of diversity techniques in such systems via appropriately designed signal processing algorithms at both the transmitters and receivers. Practical, high-performance systems require that such diversity techniques be efficient in their use of resources such as power, bandwidth, and hardware cost and that they meet often stringent computational and delay constraints.

Three main forms of diversity are traditionally exploited in communication systems for fading channels: temporal, spectral, and spatial diversity.

Temporal diversity is effective when the fading is timeselective, i.e., fluctuates with time. The degree to which

Manuscript received December 22, 1995; revised August 21, 1996. This work was supported in part by the Advanced Research Projects Agency monitored by ONR under Contract N00014-93-1-0686, the National Science Foundation under Grants MIP-9502885 and NCR-9457509, and the Office of Naval Research under Grant N00014-96-1-0930.

The authors are with the Department of Electrical Engineering and Computer Science, Massachusetts Institute of Technology, Cambridge, MA 02139 USA.

Publisher Item Identifier S 1053-587X(97)00530-8. this form of diversity can be exploited depends on delay constraints in the system relative to the coherence time of the fading process, which, in turn, is a function of, e.g., vehicle speeds in mobile applications. These constraints are often quite stringent for two-way voice communication but can, in principle, be significantly milder for broadcast applications. Error-correction coding [1] combined with interleaving, or precoding techniques of the type described in [2] [3], are examples of ways in which temporal diversity can be efficiently exploited.

Spectral diversity is effective when the fading is frequencyselective, i.e., varies as a function of frequency. This form of diversity can be exploited when the available bandwidth for transmission is large enough that individual multipath components can begin to be resolved. Examples of systems that take advantage of frequency diversity are direct-sequence or frequency-hopped spread-spectrum communication systems, which are designed to use wideband transmission formats.

Even in situations where the fading channel is nonselective, i.e., neither time selective nor frequency selective, or when system constraints preclude the use of these forms of temporal or spectral diversity, spatial diversity can be used to provide substantial improvement in system performance. Spatial diversity involves the use of multiple antennas sufficiently well-separated at the receiver and/or the transmitter that the individual transmission paths experience effectively independent fading. The extent to which this form of diversity can be exploited depends on issues such as cost and physical size constraints.

The use of multiple antennas at the receiver, which is referred to as receive diversity, is fairly easily exploited. In essence, multiple copies of the transmitted stream are received, which can be efficiently combined using the appropriate matched filter, i.e., maximal-ratio combining [4]. As the number of antennas increases, the outage probability is driven to zero, and the effective channel approaches an additive Gaussian noise channel, which simplifies communication. However, receive diversity can be impractical in a number of applications such as broadcasting or forward-link (base-tomobile) transmission in cellular systems. In such scenarios, the use of multiple antennas at the transmitter, which is referred to as transmit diversity, is significantly more attractive.

Transmit diversity is, in general, less straightforward to exploit, particularly when bandwidth expansion is not feasible and when there is no feedback path to provide the transmitter 


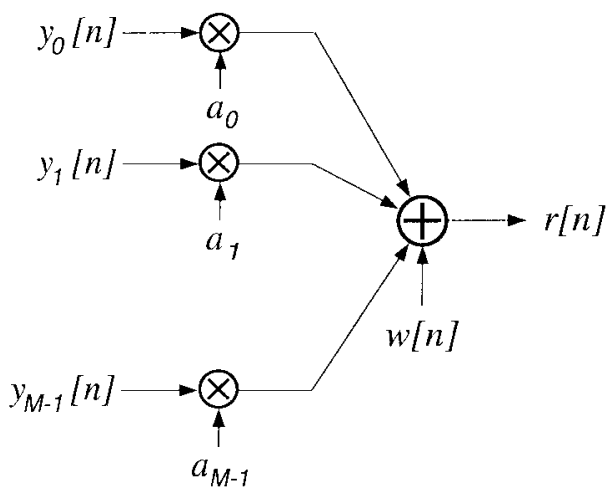

Fig. 1. Multiple-antenna nonselective Rayleigh fading channel.

with knowledge of the channel parameters. ${ }^{1}$ Nevertheless, we show in this paper that practical bandwidth-efficient techniques for exploiting transmit diversity can be developed and that they dramatically improve system performance. In particular, we develop a class of highly efficient linear signal processing algorithms for exploiting transmit diversity on nonselective fading channels without incurring bandwidth expansion. Moreover, these algorithms can be efficiently combined with other forms of diversity and error-correction coding to further improve system performance.

Our framework is also a natural and convenient one for analyzing and relating a variety of novel, bandwidth-efficient linear transmit diversity strategies that have been proposed in the literature. These include the work of Wittneben [6] and Winters [7] and that of Hiroike et al. [8] and Weerackody [9]. Finally, other aspects of the framework and the associated strategies, including information-theoretic issues, are developed in the companion work [10], [11].

An outline of the paper is as follows. In Section II, we describe the class of channels of interest, and in Section III, we describe the basic linear transmit diversity structure that is developed for such channels. In Section IV, we explore the characteristics and design of such transmit diversity schemes based on specifically linear time-invariant antenna processing. In Section V, we show that generalizing the strategy to allow linear periodically time-varying processing leads to important advantages both in terms of performance and implementation. Section VI contains some concluding remarks.

\section{Channel Model}

In our development, we focus on equivalent discrete-time baseband models of the passband channels both because they are conceptually and analytically convenient and because they lead naturally to transmitter and receiver algorithms having efficient implementations on digital signal processor (DSP) based architectures.

In particular, we consider a system with $M$ transmit antennas depicted in Fig. 1, where the (generally complex-valued) transmission from the $m$ th antenna we denote using $y_{m}[n]$ for

\footnotetext{
${ }^{1}$ Classical techniques that can be used when significant bandwidth expansion is acceptable are described in, e.g., [4]. Likewise, see, e.g., [5] for examples of transmit diversity techniques that exploit the availability of a feedback path. However, note that feedback cannot generally be exploited in applications such as broadcasting.
}

$m=0,1, \ldots, M-1$. At the receiver, we obtain

$$
r[n]=w[n]+\sum_{m=0}^{M-1} a_{m} y_{m}[n]
$$

where $w[n]$ denotes the receiver noise, which is complexvalued, zero-mean, white circular Gaussian noise with variance $\mathcal{N}_{0}$. Given sufficient physical separation among the constituent antennas, the fading coefficients $a_{0}, a_{1}, \ldots, a_{M-1}$ can be modeled as mutually independent, complex-valued, zero-mean circular Gaussian random variables with variance $\sigma_{a}^{2}$.

This nonselective fading model is generally applicable to systems in which any time variations in the channel are very slow relative to the symbol duration, and frequency variations are on scales much larger than the system bandwidth. As such this model is applicable to narrowband channels where the delay spread is smaller than the symbol duration. ${ }^{2}$ Although it is not developed in this paper, the generalization of our results to frequency selective channels is straightforward and yields the additional spectral diversity benefit one would anticipate.

In addition, we assume that the fading coefficients $a_{m}$, which are independent of the noise $w[n]$, are not known at the transmitter but are known at the receiver. Of course, perfect channel knowledge at the receiver is impossible, and in general, channel identification must be addressed in conjunction with diversity exploitation. However, the two sets of issues are largely separable, at least for the moderately slowly varying channels that arise in land-mobile radio and related applications. In practice, the receiver can obtain estimates of the channel coefficients via a trained or blind algorithm. While a detailed investigation of channel measurement algorithms and of the impact of imperfect channel identification is beyond the scope of this paper, we emphasize at the outset that we will restrict our attention to diversity techniques that do not display the sensitivity to small inaccuracies in channel measurement exhibited by, e.g., many coherent combining (beamforming) strategies.

\section{Linear Antenna PRECoding}

The information-theoretic capacity of the spatially selective fading channel in Section II can, in principle, be approached arbitrarily closely through the use of a suitably designed coding scheme [10]. In particular, a set of nonlinear systems, one for each antenna, would be designed for mapping the bit stream to the set of coded symbol streams $y_{0}[n], y_{1}[n], \ldots, y_{M-1}[n]$ at the transmitter. At the receiver, an associated nonlinear system would decode the bit stream from the received signal.

In practice, the computational complexity required to achieve rates near this capacity is prohibitive; therefore, the complexity of the transmitter and receiver are generally constrained. In this paper, we constrain complexity by considering a transmitter structure in which the bit stream is first processed by a single, suitably-designed error-correcting coder. The resulting coded symbol stream is then processed by a linear processor at each of the constituent antennas of

\footnotetext{
${ }^{2}$ Such is the case in indoor applications when the bandwidth is less than about $200 \mathrm{kHz}$ and outdoor applications when the bandwidth is less than about $20 \mathrm{kHz}$.
} 


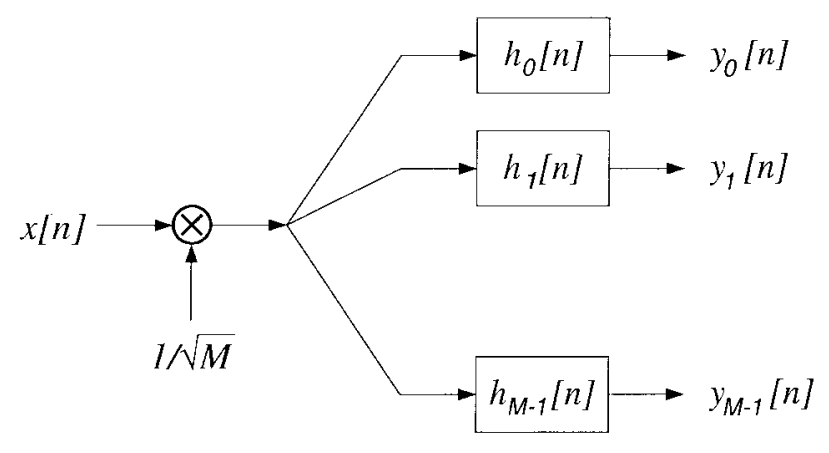

Fig. 2. Linear time-invariant antenna precoding.

the transmitter; we refer to this second stage of processing as "linear antenna precoding." This transmitter constraint leads to a dramatic reduction in system complexity while incurring a modest cost in terms of system capacity [10].

We also impose additional complexity constraints on the receiver. In particular, we consider a structure whereby the received signal is first processed by a linear equalizer, after which it is processed by a decoder appropriately matched to the coder used at the transmitter. As we will see, this additional constraint makes for further attractive complexity-capacity tradeoffs, particularly when large numbers of antennas are involved.

In the sequel, we use $x[n]$ to denote the coded symbol stream that is supplied to the linear antenna precoder at the transmitter. In turn, at the receiver, the linear equalizer processes $r[n]$ to obtain $\hat{x}[n]$ - in effect, estimates of the coded symbols - which are subsequently decoded.

\section{Linear Time-InVARIANT ANTENNA PRECODING}

When the linear antenna precoding takes the form of specifically linear time-invariant (LTI) filtering, as will be our focus in this section, the resulting precoder takes the form depicted in Fig. 2. We use $h_{m}[n]$ to denote the (generally complexvalued) unit-sample response of the filter associated with the $m$ th antenna and refer to this as a "signature" of the precoder. In turn, the collection of $M$ signatures is referred to as the signature set. With this notation, we have, via (1),

$$
y_{m}[n]=\frac{1}{\sqrt{M}} \sum_{n=-\infty}^{+\infty} h_{m}[n] x[n-k] .
$$

The associated Fourier transform of each signature will be denoted by

$$
H_{m}(\omega)=\sum_{n} h_{m}[n] e^{-j \omega n} .
$$

Of interest will be the behavior of such transmit diversity schemes for different antenna array sizes $M$. Accordingly, for future convenience, we refer to a collection of signature sets $\left\{h_{m}[n]\right\}_{m=0}^{M-1}$ indexed by $M$ as a family of signature sets. Although our signature notation does not indicate the associated value of parameter $M$, this will generally be clear from context.
In anticipation of our subsequent development, we restrict our attention to families of signature sets with some convenient characteristics. We term such signature sets admissible.

Definition 1: A family of signature sets is termed admissible if the following conditions are satisfied:

$$
\begin{gathered}
\frac{1}{M} \sum_{m=0}^{M-1}\left|H_{m}(\omega)\right|^{2}=1, \quad \text { all } \omega \\
\rho_{M}(\omega, \nu)=\frac{1}{M} \sum_{m=0}^{M-1} H_{m}(\omega) H_{m}^{*}(\nu) \rightarrow 0, \\
\text { as } M \rightarrow \infty \text { when } \omega \neq \nu
\end{gathered}
$$

where it is sufficient for the convergence in (4b) to be pointwise.

Condition (4a) is a natural one; it ensures that the total average transmitted power is independent of $M$. In particular, the transmitted power at the $m$ th antenna is

$$
\operatorname{var} y_{m}[n]=\frac{1}{2 \pi M} \int_{-\pi}^{\pi}\left|H_{m}(\omega)\right|^{2} S_{x x}(\omega) d \omega
$$

where $S_{x x}(\omega)$ is the power spectrum of the symbol stream $x[n]$. Hence, the total transmitted power is

$$
\begin{aligned}
& \sum_{m=0}^{M-1} \operatorname{var} y_{m}[n]=\frac{1}{2 \pi} \int_{-\pi}^{\pi}\left[\frac{1}{M} \sum_{m=0}^{M-1}\left|H_{m}(\omega)\right|^{2}\right] S_{x x}(\omega) d \omega \\
& =\frac{1}{2 \pi} \int_{-\pi}^{\pi} S_{x x}(\omega) d \omega=\operatorname{var} x[n]
\end{aligned}
$$

where the second equality follows from (4a). Condition (4b) is less transparent at this stage; it will ensure that certain attractive asymptotic characteristics can be achieved.

It is straightforward to construct examples of systems that possess these properties. For instance, a natural approach to obtaining transmit antenna diversity is to assign each antenna a distinct portion of the available bandwidth. In terms of our framework, this corresponds to the use of antenna signatures with ideal bandpass characteristics, i.e.,

$$
H_{m}(\omega)=\left\{\begin{array}{cc}
\sqrt{M} & m \pi / M<|\omega|<(m+1) \pi / M \\
0 & \text { elsewhere in }|\omega|<\pi
\end{array}\right.
$$

That the condition (4a) is met with these signatures is immediately apparent. To verify (4b), it suffices to note that for any distinct $\omega$ and $\nu, \rho_{M}(\omega, \nu)=0$ for all $M>\pi /|| \omega|-| \nu||$.

The ideal bandpass signatures are infinite length and, furthermore, unrealizable. However, families of practical finitelength signatures with the desired characteristics can be readily constructed. To develop this result, first note that for signatures $h_{m}[n]$ of length $M$, we can construct the matrix representation

$$
\mathbf{H}=\left[\begin{array}{cccc}
h_{0}[0] & h_{0}[1] & \cdots & h_{0}[M-1] \\
h_{1}[0] & h_{1}[1] & \cdots & h_{1}[M-1] \\
\vdots & \vdots & \ddots & \vdots \\
h_{M-1}[0] & h_{M-1}[1] & \cdots & h_{M-1}[M-1]
\end{array}\right] .
$$

For such signatures, we have the following theorem, whose straightforward proof is contained in Appendix A. 
Theorem 1: For a family of signature sets whose constituent signatures have length $M$ to be admissible in the sense of Definition 1 , it is sufficient that $\mathbf{H}$ in (5) be a unitary matrix, i.e., that each signature set consist of orthogonal signatures.

From Theorem 1, we see that we can conveniently choose an arbitrary unitary matrix $\mathbf{H}$ to construct our signature set. For example, when we choose $\mathbf{H}=\mathbf{I}$, where $\mathbf{I}$ is the $M \times M$ identity matrix so that $h_{m}[n]=\delta[n-m]$, we obtain a scheme explored both by Wittneben [6] for the case $M=2$ and, more generally, by Winters [7]. In these schemes, each antenna transmits a delayed copy of the sequence $x[n]$. We can also choose $\mathbf{H}=\mathbf{F}$ or $\mathbf{H}=\boldsymbol{\Xi}$, which are the discrete Fourier transform (DFT) and Hadamard matrices, respectively. ${ }^{3}$ The former corresponds to a generally complex-valued signature set and can be viewed as a finite length variant of the frequency band allocation example described earlier. However, for $M=2$, the DFT- and Hadamard-based schemes specialize to a common scheme that is also explored by Wittneben [6].

Note too that for the choice $\mathbf{H}=\mathbf{I}$, the transmitted signals $y_{m}[n]$ have the same amplitude characteristic (i.e., marginal probability density function) as $x[n]$ regardless of $M$. By contrast, for $\mathbf{H}=\boldsymbol{\Xi}$, the amplitude distribution for each $y_{m}[n]$ approaches a Gaussian for $M \rightarrow \infty$. Hence, for large $M$, the peak-to-average power requirements can be demanding. Moreover, all transmit diversity schemes of the type we have described have the property that the signal component of the received waveform has an amplitude distribution that is asymptotically Gaussian due to the superposition of the multiple transmissions. This property may be attractive from certain transmission security perspectives.

\section{A. System Characteristics and Receiver Design}

Linear antenna precoding has a powerful interpretation as a channel transformation strategy. To see this, observe that by specializing (1), the received signal can be expressed in the form

$$
r[n]=a[n] * x[n]+w[n]
$$

where

$$
a[n]=\frac{1}{\sqrt{M}} \sum_{m=0}^{M-1} a_{m} h_{m}[n]
$$

is the unit-sample response of the "effective" channel generated by the antenna precoder. This channel has frequency response

$$
A(\omega)=\frac{1}{\sqrt{M}} \sum_{m=0}^{M-1} a_{m} H_{m}(\omega)
$$

${ }^{3}$ The DFT matrix of dimension $M$ is defined via

$$
[\mathbf{F}]_{k, l}=e^{-j 2 \pi k l / M}
$$

whereas $\boldsymbol{\Xi}_{M}$, which is the Hadamard matrix of dimension $M$, is defined recursively when $M$ is a power of two: For $M=2,4, \ldots$,

$$
\boldsymbol{\Xi}_{M}=\frac{1}{\sqrt{2}}\left[\begin{array}{cc}
\boldsymbol{\Xi}_{M / 2} & \boldsymbol{\Xi}_{M / 2} \\
\boldsymbol{\Xi}_{M / 2} & -\boldsymbol{\Xi}_{M / 2}
\end{array}\right]
$$

where $\boldsymbol{\Xi}_{1}=1$ which is a zero-mean, $2 \pi$-periodic, Gaussian random process in frequency $\omega$, with variance $\sigma_{a}^{2}$. Hence, we see that the antenna precoding effectively transforms the original nonselective fading channel into a frequency-selective fading channel.

This key observation has important implications for receiver design. In particular, it implies that any of a variety of traditional approaches to decoding in the presence of intersymbol interference can be exploited. Examples include maximum likelihood sequence detection, decision feedback equalization, or linear equalization [12]. When suitably designed, such receivers can exploit this inherent frequency diversity to substantially improve system performance.

It is also important to note that the frequency selective channel synthesized via antenna precoding has some properties that naturally occurring frequency selective channels typically do not. For example, choosing precoding signatures $h_{m}[n]$ to meet the conditions of Definition 1 ensures that the resulting frequency diversity has strong asymptotic mixing characteristics, which allow a more substantial diversity benefit to be realized. In addition, the channel identification problem that must be addressed in practice is substantially easier in our case than for naturally occurring frequency-selective channels due to the way in which this channel is parameterized.

In this section, we restrict our attention to linear equalizers, which remain practical even when the number of antennas is large, and follow the equalizer with a decoder. Specifically, we consider an LTI equalizer with unit-sample response $b[n]$ so that the equalized signal is

$$
\hat{x}[n]=b[n] * r[n]=\sum_{k} b[k] r[n-k] .
$$

In general, since the receiver knows the fading coefficients, the equalizer $b[n]$ will depend on the channel response $a[n]$. For example, one possible choice for the equalizer is the matched filter, for which $b[n]=a[-n]$. This is equivalent to a traditional RAKE receiver, which is often used to exploit frequency diversity [13]. However, we will see that other equalizers have significant advantages in our application.

In the sequel, we will develop some important system properties that result when any of a broad range of linear equalizers is used. To allow for precise results, we impose some relatively mild restrictions on the class of equalizers under consideration. Specifically, we have the following definition.

Definition 2: Let $b[n]$ be the unit-sample response of an LTI equalizer with frequency response $B(\omega)$. Then, $b[n]$ is an admissible equalizer if $B(\omega)$ can be written in the form

$$
B(\omega)=f(A(\omega))
$$

where $A(\omega)$ is as defined in (8), and where $f(\cdot)$ is a complexvalued function that satisfies the admissibility conditions:

1) $f(\cdot)$ is bounded within any finite region of the complex plane, i.e., for every $R$, there exists a positive real constant $M(R)<\infty$ such that

$$
|f(z)|<M(R) \quad \text { for all }|z|<R .
$$


2) $f(\cdot)$ grows more slowly than a quadratic exponential, i.e., ${ }^{4}$

$$
f(z) \sim o\left(e^{\varepsilon|z|^{2}}\right) \quad \text { for every } \varepsilon>0 .
$$

For any LTI equalizer $b[n]$, we have that

$$
\hat{x}[n]=b[n] * a[n] * x[n]+b[n] * w[n]
$$

so that

$$
C(\omega)=A(\omega) B(\omega)
$$

is the effective frequency response after equalization. Note that if $B(\omega)$ is an admissible equalizer, then both $B(\omega)$ and $C(\omega)$ have means and variances that are not only finite but, via (10), are also functions only of $\sigma_{a}^{2}$ and not of $\omega$. Accordingly, we denote these quantities using

$$
\begin{array}{cl}
\mu_{b}=E[B(\omega)] & \mu_{c}=E[C(\omega)] \\
\sigma_{b}^{2}=\operatorname{var} B(\omega) & \sigma_{c}^{2}=\operatorname{var} C(\omega) .
\end{array}
$$

In turn, we obtain the following conceptually appealing theorem that characterizes the composite system consisting of the antenna precoder, channel, and equalizer. A proof is provided in Appendix B.

Theorem 2: Let $x[n]$ be a sequence of zero-mean uncorrelated symbols, each with energy $\mathcal{E}_{s}$. Furthermore, for every $M$, let $a_{m}$ for $m=0,1, \ldots, M-1$ be a collection of independent zero-mean complex-valued Gaussian random variables with variance $\sigma_{a}^{2}$. Finally, suppose $b[n]$ is the unit-sample response of an admissible equalizer in the sense of Definition 2 and that the antenna precoder signature sequences $h_{m}[n]$ satisfy the conditions (4). Then, as $M \rightarrow \infty$, we have that $\hat{x}[n]$ defined via (11) with (7) satisfies, for each $n,{ }^{5}$

$$
\hat{x}[n] \stackrel{\mathrm{m} . \mathrm{s}}{\longrightarrow} \mu_{c} x[n]+v[n]
$$

where $v[n]$ is a complex-valued, marginally Gaussian, zeromean white noise sequence, uncorrelated with the input symbol stream $x[n]$, and having variance

$$
\operatorname{var} v[n]=\mathcal{E}_{s} \sigma_{c}^{2}+\mathcal{N}_{0}\left(\sigma_{b}^{2}+\left|\mu_{b}\right|^{2}\right) .
$$

Theorem 2 asserts that given transmit antenna diversity of this form, the channel "seen" by the coded symbol stream is transformed from a fading channel into a marginally Gaussian white noise channel. From (13), we see there are two components in this equivalent noise: One component is due to the original receiver noise, whereas the second is due to intersymbol interference (ISI) generated by the transmit diversity and, hence, has a variance that scales with the symbol energy. In effect, we see that this ISI is transformed into a comparatively more benign form of uncorrelated, additive noise. Furthermore, while Theorem 2 establishes only an

\footnotetext{
${ }^{4}$ The order notation $o(\cdot)$ is to be interpreted in the usual sense. If $p(z) \sim$ $o(q(z))$, then
}

$$
\lim _{|z| \rightarrow \infty} \frac{p(z)}{q(z)}=0
$$

${ }^{5}$ We use the notation $\stackrel{\mathrm{m} . \mathrm{s}}{\longrightarrow}$ to denote convergence in the mean-square sense. asymptotic result, we will see that the equivalent quasiGaussian channel is a useful model even when the number of antennas $M$ used is finite.

In effect, Theorem 2 implies that using an increasingly large number of transmit antennas reduces variance in system performance. To appreciate what this means in practice, it is useful to consider a scenario in which there are a collection of suitably spaced receivers all at roughly the same radius from the transmitter. With a single transmit antenna, the signal-tonoise ratio (SNR) of the transmitted data as measured at a receiver will vary from receiver to receiver. However, when transmit diversity via a multiple antenna cluster is exploited, this variation from receiver to receiver is reduced. Moreover, our asymptotic result establishes that using a large number of such transmit antennas, variation from receiver to receiver is effectively eliminated. In other words, all receivers "see" the data at effectively the same SNR. This property can also be interpreted as meaning that the outage probability as it is usually defined (i.e., the probability that the SNR drops below some prescribed threshold at a receiver) is driven to zero as the number of antennas is increased.

As another interpretation, this theorem can also be viewed as the transmit diversity counterpart of an analogous result established for a class of bandwidth-efficient temporal diversity schemes in [2] and [3]. As a result, many of the comments that apply in the temporal diversity scenario have counterparts in this transmit diversity case. For example, we stress that Theorem 2 focuses primarily on the second-moment characteristics of the equivalent model. In particular, the noise $v[n]$ is not, in general, a Gaussian process-only marginally so. Hence, that the samples $v[n]$ are uncorrelated does not imply they are independent. Likewise, while $x[n]$ and $v[n]$ are uncorrelated, there are invariably higher order statistical dependencies between these sequences.

The utility of the equivalent model lies in the fact that it suggests some powerful and computationally highly efficient receiver structures. In particular, in the absence of additional coding, good performance can be maintained if computationally expensive, Viterbi-algorithm-based maximum likelihood sequence detection of the type described by Winters [7] is replaced with simple symbol-by-symbol detection, as would be used on a true additive white Gaussian noise channel. Even more significantly, such simplifications mean that it is practical to combine this antenna diversity with additional error-correction coding for the Gaussian channel, as suggested earlier. For example, if trellis coding is used, the usual Viterbi decoding can be applied after equalization as if the channel were Gaussian. ${ }^{6}$

In general, system performance will depend substantially on the particular equalizer chosen for the system. Indeed, the SNR associated with the equivalent channel of Theorem 2 depends strongly on the choice of equalizer. Consequently, a useful criterion for equalizer design is to select among admissible equalizers that yielding the largest SNR in the equivalent

\footnotetext{
${ }^{6}$ The price we pay for such substantial computational advantages of this strategy can be measured in terms of the usual performance limitations inherent in the use of linear equalizers; for a discussion of such losses, see, e.g., [12].
} 
channel. Conveniently, when $b[n]$ is admissible, the SNR in the equivalent channel follows immediately from Theorem 2 as

$$
\begin{aligned}
\gamma(B) & =\frac{\left|\mu_{c}\right|^{2}}{\sigma_{c}^{2}+\xi_{0}\left(\sigma_{b}^{2}+\left|\mu_{b}\right|^{2}\right)} \\
= & \frac{|E[A(\omega) B(\omega)]|^{2}}{\operatorname{var}[A(\omega) B(\omega)]+\xi_{0} E\left[|B(\omega)|^{2}\right]}
\end{aligned}
$$

where

$$
\xi_{0}=\mathcal{N}_{0} / \mathcal{E}_{s} .
$$

The resulting optimization problem is then directly analogous to one that arose in [2] and [3], and the solution, which follows from a relatively straightforward application of the Cauchy-Schwarz inequality, is given by

$$
B(\omega) \propto \frac{A^{*}(\omega)}{|A(\omega)|^{2}+\xi_{0}}
$$

where the constant of proportionality is arbitrary.

The optimum equalizer has some useful interpretations. First, this equalizer not only maximizes the equivalent channel SNR but also, when suitably normalized, makes $\hat{x}[n]$ a minimum mean-square error linear estimate of $x[n]$. This is appealing from the point of view of implementation since it suggests that adaptive equalizers based on least-mean-square (LMS) or recursive least-squares (RLS) algorithms can be used in practice. As a second interpretation, we note that the numerator of (16) is a conventional matched filter (i.e., RAKE receiver); therefore, the denominator can be viewed as an additional compensation stage that takes into account the special characteristics of the equivalent noise in this context, as discussed earlier.

As a final comment, we emphasize that, in effect, this section has identified a broad class of transmit diversity strategies having the characteristic that the variance in performance from receiver to receiver is driven to zero as the number of antennas is increased. Within this class, through optimization of the equalizer, we have identified those strategies having, asymptotically and simultaneously, the best possible average performance. Moreover, as we will see in the next section, the use of such transmit diversity leads, in fact, to a substantial increase in average performance over that in single antenna systems.

\section{B. Performance}

In this section, we develop useful closed-form bounds on the performance of our transmit diversity system with finitely many antennas $M$ by examining asymptotic behavior. Furthermore, the bounds we obtain are asymptotically tight and allow us to quantify the performance that is achievable in practice using massive (i.e., very large $M$ ) transmit antenna clusters. Additional aspects of the performance of the schemes for finite $M$ are developed later in Section V-D.

The asymptotic $(M \rightarrow \infty)$ performance characteristics of the optimized transmit diversity system can be conveniently developed by further exploiting the common form of the equivalent quasi-Gaussian channel generated by our transmit diversity scheme and that generated by temporal diversity via spread-response precoding in [2] and [3].

Specifically, while the SNR in the original channel, i.e.,

$$
\alpha_{0}(\omega)=\frac{|A(\omega)|^{2} \mathcal{E}_{s}}{\mathcal{N}_{0}}
$$

is both random and varies as a function of frequency $\omega$, with the optimum equalizer, i.e., (16), we immediately obtain that the SNR in the asymptotic equivalent channel is a deterministic constant of the form

$$
\gamma_{0}=\frac{1}{E\left[\frac{1}{\alpha_{0}+1}\right]}-1=\frac{1}{\zeta_{0} e^{\zeta_{0}} E_{1}\left(\zeta_{0}\right)}-1
$$

where

$$
1 / \zeta_{0}=E\left[\alpha_{0}(\omega)\right]=\frac{\sigma_{a}^{2} \mathcal{E}_{s}}{\mathcal{N}_{0}}
$$

is the average SNR in the original channel, and where $E_{1}(\cdot)$ denotes the exponential integral [14] defined via

$$
E_{1}(\nu)=\int_{\nu}^{\infty} \frac{e^{-t}}{t} d t .
$$

A potentially useful estimate of capacity for our transmit diversity scheme results when we ignore the higher order statistical dependencies in the equivalent channel of Theorem 2 and view the channel as strictly Gaussian channel. In this case, the mutual information between $\hat{x}[n]$ and $x[n]$ when $x[n]$ is Gaussian is

$$
\mathcal{C}=\log \left(1+\gamma_{0}\right)=-\log \left(\zeta_{0} e^{\zeta_{0}} E_{1}\left(\zeta_{0}\right)\right)
$$

which suggests bit rates that may be achievable when sufficiently extensive additional coding is applied to the bit stream before precoding at the antennas.

As is developed in [10], this effective capacity can be compared with that of related transmit diversity systems without such stringent computational constraints. For example, removing the constraint that the front end of our receiver be a linear equalizer and allowing an arbitrarily complex decoder leads to an increase in capacity. Specifically, with no receiver constraint, the capacity of the asymptotic $(M \rightarrow \infty)$ transmitantenna diversity channel using linear antenna precoding with signatures $h_{m}[n]=\delta[n-m]$ takes the form [10]

$$
\mathcal{C}_{\mathrm{L}}=E\left[\log \left(1+\alpha_{0}\right)\right]=e^{\zeta_{0}} E_{1}\left(\zeta_{0}\right) .
$$

If, in addition, we remove the constraint that the antenna precoding be linear and allow an arbitrarily complex encoder at each antenna, then still higher capacities are achievable. In particular, the corresponding asymptotic $(M \rightarrow \infty)$ transmitdiversity channel has capacity [10]

$$
\mathcal{C}_{\mathrm{T}}=\log \left(1+E\left[\alpha_{0}\right]\right)=\log \left(1+1 / \zeta_{0}\right)
$$

which is equivalent to the capacity of an additive white Gaussian noise channel, and can be compared with the capacity associated with unconstrained receive diversity [11]. These 


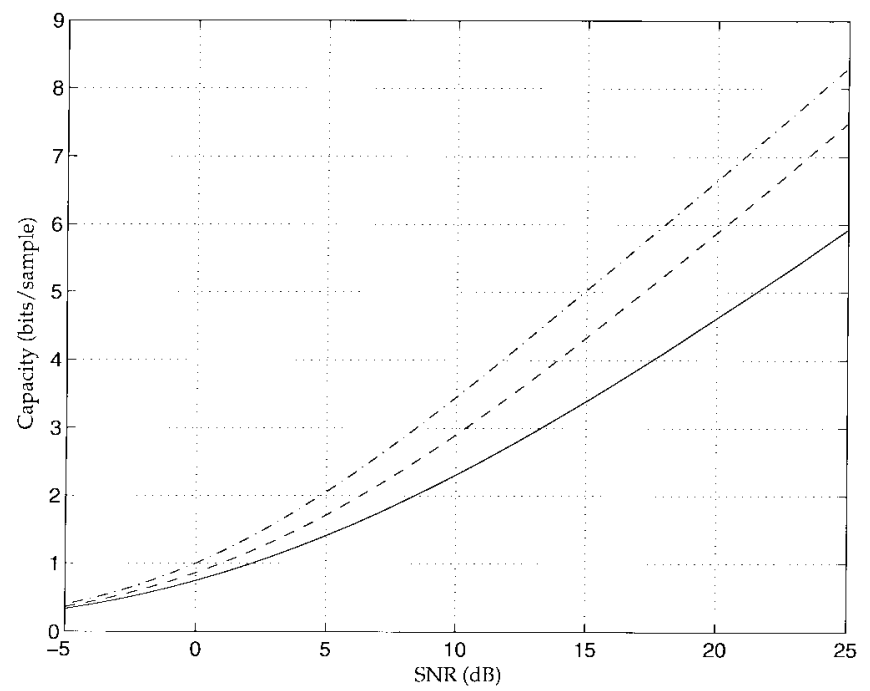

Fig. 3. Rayleigh fading channel capacities with infinite transmit diversity. The solid curve is the capacity estimate $\mathcal{C}$ corresponding to the use of linear antenna precoding at the transmitter and linear equalization at the receiver. The dashed curve indicates the capacity $\mathcal{C}_{\mathrm{L}}$ when linear antenna precoding is used but there are no receiver constraints. Finally, the dash-dotted curve is the transmit diversity capacity $\mathcal{C}_{\mathrm{T}}$ when there are no transmitter or receiver constraints.

capacities are depicted in Fig. 3 as a function of average SNR in bit per sample.

When augmenting linear antenna precoding with coding to approach capacity, the quasi-Gaussian equivalent channel model suggests that any of the traditional forms of coding for the bandlimited additive white Gaussian noise channel would be appropriate. In particular, as we suggested earlier, conventional implementations of trellis-coded modulation appear to be well suited to this application. Although achievement of rates near capacity requires codes with very high constraint lengths, short codes can be used in practice to achieve more modest performance enhancements. In fact, even with no additional coding, the use of antenna precoding leads to substantially reduced bit-error rates over systems without such transmit diversity.

As an illustration, consider the case in which $x[n]$ is an uncoded quadrature phase-shift keying (QPSK) symbol stream. With infinite transmit diversity in the form of antenna precoding, the average bit error probability is

$$
\mathcal{P}=\mathcal{Q}\left(\sqrt{\gamma_{0}}\right)
$$

where

$$
\mathcal{Q}(\nu)=\frac{1}{\sqrt{2 \pi}} \int_{\nu}^{\infty} e^{-t^{2} / 2} d t
$$

and where $\gamma_{0}$ is as defined in (18) with (19).

For comparison, without transmit diversity, the average QPSK bit error probability is [13]

$$
\mathcal{P}_{0}=\frac{1}{2}\left(1-\frac{1}{\sqrt{2 \zeta_{0}+1}}\right)
$$

while with infinite (normalized) receive diversity and maximal ratio combining, it is

$$
\mathcal{P}_{\mathrm{R}}=\mathcal{Q}\left(1 / \sqrt{\zeta_{0}}\right)
$$

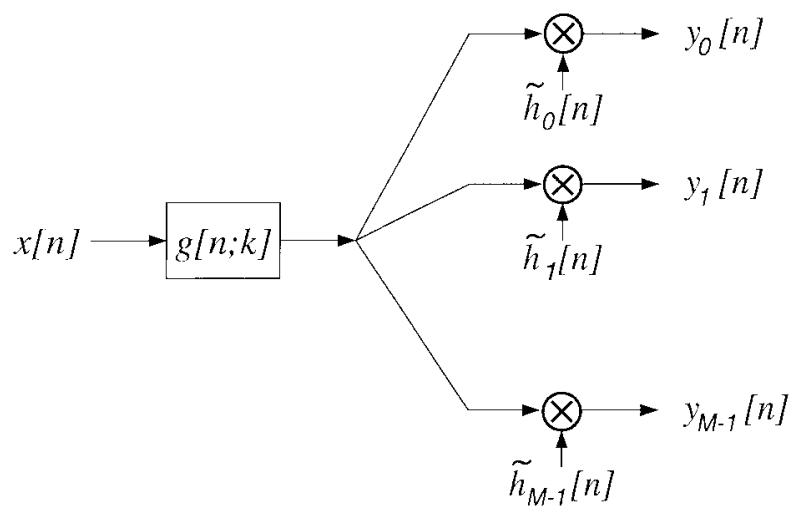

Fig. 4. Linear periodically time-varying antenna precoding structure.

At high SNR (i.e., small $\zeta_{0}$ ), the bit error rate with infinite transmit diversity via antenna precoding is given by

$$
\mathcal{P} \sim \sqrt{\frac{\log \left(1 / \zeta_{0}\right)}{1 / \zeta_{0}}} \exp \left(-\frac{1 /\left(2 \zeta_{0}\right)}{\log \left(1 / \zeta_{0}\right)}\right),
$$

whereas that with infinite receive diversity is given by

$$
\mathcal{P}_{\mathrm{R}} \sim \sqrt{\zeta_{0}} e^{-1 /\left(2 \zeta_{0}\right)} .
$$

Comparing (26) with (27), we see that both fall off faster than any power of SNR and that the difference amounts to a logtype penalty in the transmit case. In principle, these results can also be compared with the achievable uncoded bit error rate $\mathcal{P}_{\mathrm{L}}$ when the optimum unconstrained receiver is employed. This bit error rate was explored by Winters [7] for the choice of signature set corresponding to $h_{m}[n]=\delta[n-m]$. In this case, the optimum nonlinear receiver implementing maximum likelihood sequence detection has a complexity that grows exponentially with the number of antennas, in contrast to the linear growth in complexity when our receivers based on linear equalizers are used.

As we indicated at the outset, the asymptotic bit error rate results we have described provide useful bounds on what can be obtained in practice using these methods with finite transmit diversity $(M<\infty)$. However, the LTI antenna precoding we have developed thus far is somewhat impractical to implement. The problem arises because even with a finite number of antennas, the optimum equalizer has, in general, an infinite length, unrealizable unit-sample response. In addition, while finite-length approximations can give reasonable performance in practice, they do so at a cost of excessive delay. Fortunately, these problems can be conveniently circumvented through a generalization of the basic linear antenna precoding strategy, which we develop next.

\section{LINEAR PERIODICALLY TIME-VARYING ANTENNA PRECODING}

In this section, we consider a case in which the processing at each antenna takes the form of more general linear periodically time-variant (LPTV) filtering. We restrict our attention to a particular class of such systems that admit the factorization depicted in Fig. 4. In particular, the coded symbol stream $x[n]$ is first processed by a common LPTV prefilter that is time 
varying with some period ${ }^{7} K \geq 2$ and has length $M$, and whose kernel we denote by $g[n ; k]$. The result

$$
y[n]=\sum_{k} g[n ; k] x[n-k]
$$

is then subsequently processed at each of the antennas. Specifically, this prefiltered stream is modulated at each antenna by a different $M$-periodic sequence, i.e.,

$$
y_{m}[n]=\tilde{h}_{m}[n] y[n]
$$

where $\widetilde{h}_{m}[n]$ is the generally complex-valued periodic sequence associated with the $m$ th antenna. We use $h_{m}[n]$ to denote a single period of this modulating sequence, i.e.,

$$
h_{m}[n]=\left\{\begin{array}{cc}
\tilde{h}_{m}[n] & 0 \leq n \leq M-1 \\
0 & \text { otherwise }
\end{array}\right.
$$

and refer to this as the "signature" of the associated antenna. ${ }^{8}$ We first consider the design of the signatures $h_{m}[n]$ and then turn our attention to the design of the prefilter kernel $g[n ; k]$.

\section{A. Signature Design}

To design suitable signature sequences, we begin by considering the response of channel to the prefiltered symbol stream $y[n]$. For this subsystem, we have, using (29) in (1), that

$$
r[n]=w[n]+\tilde{a}[n] y[n]
$$

where

$$
\tilde{a}[n]=\sum_{m=0}^{M-1} a_{m} \tilde{h}_{m}[n]
$$

is an $M$-periodic fading sequence. For future convenience, we denote one period of this sequence using $a[n]$, specifically,

$$
a[n]=\sum_{m=0}^{M-1} a_{m} h_{m}[n] .
$$

From (30), we make the important observation that the signature modulation subsystem effectively transforms the original nonselective fading channel into a time-selective one. As such, this transformation is the dual of the nonselective to frequency-selective transformation we explored in Section IV as an interpretation of LTI antenna precoding. From this perspective, we see that our prefilter should be designed to allow the inherent time diversity introduced by the modulation process to be efficiently exploited at the receiver.

The maximum time diversity benefit is obtained when the fading is independent among time samples within a period, and thus, we design our signature sequences to ensure that this condition is met. To proceed, it is convenient to again collect our signatures into a matrix of the form of (5).

From (32), we see that the coefficients $a[0], a[1], \ldots, a[M-$ 1] are zero-mean and jointly Gaussian. Moreover, the correlation between an arbitrary pair of these coefficients is

\footnotetext{
${ }^{7}$ We remark in advance that the parameter $K$ plays a relatively minor role in the development, and in fact, as will become apparent, fixing $K=2$ suffices in practice.

${ }^{8}$ Again, although the signature $h_{m}[n]$ is a function of the parameter $M$, for notational convenience, we suppress this dependence.
}

proportional to the inner product between the corresponding columns of $\mathbf{H}$ in (5), i.e.,

$$
\begin{gathered}
\operatorname{cov}(a[n], a[l])=E\left[a[n] a^{*}[l]\right] \\
\quad=\sigma_{a}^{2} \sum_{m=0}^{M-1} h_{m}[n] h_{m}^{*}[l] .
\end{gathered}
$$

Hence, the coefficients $a[0], a[1], \ldots, a[M-1]$ will be statistically independent when the columns of $\mathbf{H}$ are orthogonal.

To determine the appropriate normalization of the signatures, we impose the constraint the total transmit power be conserved. Provided the prefilter is an orthonormal transformation, as we will impose in Section V-B, we obtain, via (28), that

$$
\operatorname{var} y[n]=\operatorname{var} x[n] .
$$

Using, in order, (29) and (34), we obtain that the total transmitted power is

$$
\sum_{m=0}^{M-1} \operatorname{var} y_{m}[n]=\operatorname{var} x[n] \sum_{m=0}^{M-1}\left|h_{m}[n]\right|^{2} .
$$

Since this total transmitted power must equal var $x[n]$, we obtain the condition that the columns of $\mathbf{H}$ must have unit norm.

Hence, we see that to achieve the maximum diversity benefit and simultaneously meet our power constraint, it is necessary and sufficient that $\mathbf{H}$ must be a unitary matrix. Equivalently, it is necessary and sufficient that the signatures (i.e., rows of H) be an orthonormal set.

Evidently, there are infinitely many signature sets that ensure the independent fading condition. For example, the choice $\mathbf{H}=\mathbf{I}$ corresponds to a strategy in which prefiltered symbols are dealt among the antennas and transmitted in order. A disadvantage of this particular choice, however, is the high peak-power requirement. As an alternative, we could choose $\mathbf{H}=\mathbf{F}$, where $\mathbf{F}$ is again the suitably sized DFT matrix. In this case, the result can be interpreted as an efficient discretetime variant of the phase-sweeping transmit diversity system explored by Hiroike et al. [8] and Weerackody [9]. As a final example, the choice $\mathbf{H}=\boldsymbol{\Xi}$, where $\boldsymbol{\Xi}$ is again the Hadamard matrix, has some particularly attractive characteristics. First, like the DFT-based signatures, the Hadamard-based signatures have minimal peak power requirements. Second, since Hadamard-based signatures are binary valued, they can be implemented with very low computational complexity, requiring additions and sign changes but no multiplies.

\section{B. Prefilter Design}

We next turn our attention to the design of a LPTV prefilter that can exploit the time diversity generated by the signature modulation process. It is important to recognize at the outset that prefiltering can be avoided (i.e., we can set $g[n, k]=\delta[k]$ ), provided coding is used prior to the antenna precoding stage of the transmitter. In particular, suitably designed error correction coding can be used to achieve a substantial diversity benefit on time-selective fading channels like those we have synthesized through our antenna precoding. However, this coding, as 


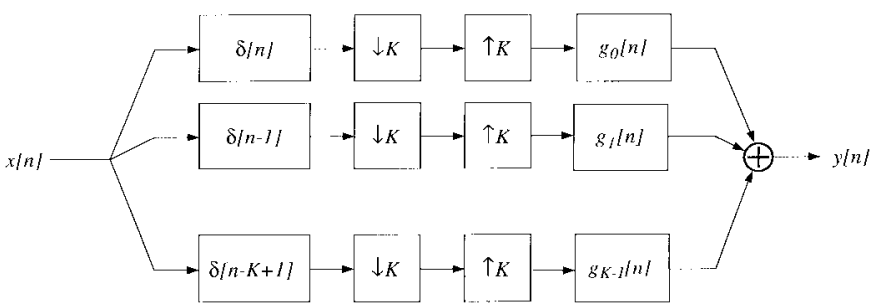

Fig. 5. Canonical realization of a linear periodically time-varying system.

well as the associated decoding that would be required at the receiver, can be computationally prohibitive in practice, particularly when the number of antennas is large.

When computation is constrained, [2] suggests that the best diversity benefit is often achieved by combining (or sometimes even replacing) coding with suitably designed prefiltering. In particular, the bandwidth-preserving LPTV spread-response precoders developed in [3] (after [2]) are naturally suited as prefilters for our application, as will become apparent. Indeed, for Rayleigh-distributed time-selective fading channels, these orthogonal systems provide, in an appropriate sense, the optimum linear diversity benefit with very low computational complexity [3].

The prefilters of interest are, specifically, the maximallyspread LPTV precoders developed in [3]. In the remainder of this section, we briefly summarize their salient characteristics for the purposes of this paper. A canonical description for a general period- $K$ LPTV system is given by the input-output relationship

$$
y[n]=\sum_{l=-\infty}^{+\infty} \sum_{i=0}^{K-1} x[l K-i] g_{i}[n-l K]
$$

which is specified in terms of a vector of $K$ unit-sample responses $^{9}$

$$
\mathbf{g}[n]=\left[\begin{array}{llll}
g_{0}[n] & g_{1}[n] & \cdots & g_{K-1}[n]
\end{array}\right]^{\mathrm{T}} .
$$

The effective length or spread of the prefilter is defined to be the length of this vector sequence $\mathbf{g}[n]$. The associated implementation for these systems is shown in Fig. 5.

The LPTV system effects an orthonormal transformation of its input samples when the vector sequence $\mathbf{g}[n]$ or, equivalently, its Fourier transform vector

$$
\begin{aligned}
& \mathbf{G}(\omega)=\sum_{n=-\infty}^{+\infty} \mathbf{g}[n] e^{-j \omega n} \\
& \triangleq\left[\begin{array}{llll}
G_{0}(\omega) & G_{1}(\omega) & \cdots & G_{K-1}(\omega)
\end{array}\right]^{\mathrm{T}}
\end{aligned}
$$

meets certain conditions. In particular, when we express the set of Fourier transforms (38) in the polyphase form

$$
\mathbf{G}(\omega)=\mathbf{Q}(K \omega) \Delta(\omega)
$$

\footnotetext{
${ }^{9}$ It is straightforward to relate this kernel notation to that in (28). The response of the system at time $n$ to a unit-sample at time $n-k$ is

$$
g[n ; k]=g_{i}[n-l K]
$$
}

\begin{tabular}{|c|c|c|c|c|c|c|c|c|}
\hline & & \\
\hline$n=$ & 0 & 1 & 2 & 3 & & 5 & 6 & 71 \\
\hline$\sqrt{8} g_{0}[n]$ & +1 & $\cdot-1$ & +1 & -1 & +1 & +1 & -1 & +1 \\
\hline$\sqrt{8} g_{1}[n]$ & +1 & +1 & +1 & -1 & -1 & -1 & +1 & -1 \\
\hline
\end{tabular}

where $l$ and $0 \leq i \leq K-1$ are uniquely defined via the relation $k=l K-i$.
TABLE I

Nonzero TAPs of the Period-2 MaXimally Spread LPTV Prefilter With SPREAd $M=8$

where $\Delta(\omega)$ is the Fourier transform of the delay chain of order $K$, i.e.,

$$
\Delta(\omega)=\left[\begin{array}{llll}
1 & e^{-j \omega} & \cdots & e^{-j \omega(K-1)}
\end{array}\right]^{\mathrm{T}}
$$

the orthogonality conditions are equivalent to the condition that the polyphase matrix $\mathbf{Q}(\omega)$ be paraunitary: ${ }^{10}$ $\mathbf{Q}^{\dagger}(\omega) \mathbf{Q}(\omega)=\mathbf{I}$.

The maximally spread prefilters are those orthogonal LPTV systems for which the unit-sample responses $g_{k}[n]$ are binary valued. A convenient construction for the associated polyphase matrix $\mathbf{Q}(\omega)$ is recursive. Specifically, we let our zeroth-order polyphase matrix be ${ }^{11}$

$$
\mathbf{Q}^{(0)}(\omega)=\boldsymbol{\Xi}
$$

Then, to obtain systems for which the spread $M$ exceeds $K$, we exploit the recursion [3]

$$
\mathbf{Q}^{(i)}(\omega)=\boldsymbol{\Xi} \boldsymbol{\Lambda}\left(M^{i-1} \omega\right) \mathbf{Q}^{(i-1)}(\omega) \quad i=1,2, \ldots
$$

where $\boldsymbol{\Lambda}(\omega)$ is the diagonal delay matrix whose diagonal is constructed from the elements of $\Delta(\omega)$, i.e., $\Lambda(\omega)=$ $\operatorname{diag} \boldsymbol{\Delta}(\omega)$ with $\boldsymbol{\Delta}(\omega)$ as defined in (40). It is straightforward to verify that the recursion (42) leads to a set of binary sequences; indeed, one can interpret (42) and (41) as implementing a particular succession of simple sequence concatenations initiated with Hadamard sequences.

Using (39), we can also express the recursion (42) directly in terms of the Fourier transform of the signature set; specifically, we have, for $i=1,2, \ldots$,

$$
\mathbf{G}^{(i)}(\omega)=\boldsymbol{\Xi} \boldsymbol{\Lambda}\left(K^{i} \omega\right) \mathbf{G}^{(i-1)}(\omega) .
$$

From (43), we can verify that the length of $\mathbf{g}[n]$ grows by a factor of $K$ with each application of the recursion so that, in particular, $\mathrm{g}^{(i)}[n]$ has spread $M=K^{i+1}$ for $i=0,1,2, \ldots$

Several sets of signature sequences obtained by the recursion (42) with (41), and corresponding to different values of $K$ and $M$, are tabulated in [3]. As one example, for $K=2$ and $M=8$, the nonzero taps of $g_{0}[n]$ and $g_{1}[n]$ are given in Table I.

\section{System Characteristics and Receiver Design}

As in the case of LTI antenna precoding, it is natural to consider using a linear equalizer at the front end of our receiver, as depicted in Fig. 6. Specifically,

$$
\hat{x}[n]=\sum_{k} g[k ; n] \hat{y}[n-k]
$$

\footnotetext{
${ }^{10}$ The superscript ${ }^{\dagger}$ denotes the conjugate-transpose operation.

${ }^{11}$ For convenience, we restrict our attention to orders $K$ for which Hadamard matrices exist. These include, for example, all integers $K$ that are powers of two.
} 


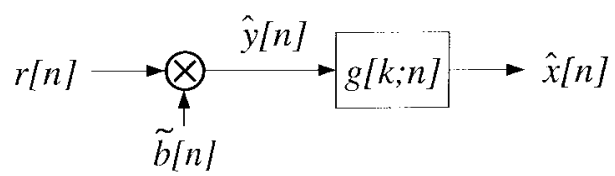

Fig. 6. Receiver structure for linear periodically time-varying antenna precoding.

where

$$
\hat{y}[n]=\tilde{b}[n] r[n]
$$

and where $\tilde{b}[n]$ is a suitable equalizer for the time-selective fading. Note that since the prefilter (with kernel $g[n ; k]$ ) is an orthogonal system, $g[k ; n]$ is the kernel of its inverse. This postfilter is conveniently implemented via the transposition of the flow graph of Fig. 5.

In developing some general system characteristics analogous to those developed in Section IV, we impose similarly mild constraints on the class of equalizers under consideration. In particular, admissible equalizers in this case take the form

$$
\tilde{b}[n]=f(\tilde{a}[n])
$$

where $f(\cdot)$ is a complex-valued function that also meets the admissibility conditions of Definition 2.

With equalizers of this type, we have that

$$
\hat{y}[n]=\tilde{c}[n] y[n]+\tilde{b}[n] w[n]
$$

where

$$
\tilde{c}[n]=\tilde{a}[n] \tilde{b}[n] .
$$

When orthogonal signatures are used as developed in Section IV-A, then

$$
E[\tilde{a}[n]]=0 \quad \operatorname{var} \tilde{a}[n]=\sigma_{a}^{2} .
$$

Hence, it follows in turn that if $\tilde{b}[n]$ is an admissible equalizer, then both $\tilde{b}[n]$ and $\tilde{c}[n]$ have means and variances that are not only finite but are also functions only of $\sigma_{a}^{2}$ and not of $n$. Accordingly, we denote these quantities using

$$
\begin{aligned}
\mu_{b} & =E[\tilde{b}[n]] & \mu_{c} & =E[\widetilde{c}[n]] \\
\sigma_{b}^{2} & =\operatorname{var} \tilde{b}[n] & \sigma_{c}^{2} & =\operatorname{var} \tilde{c}[n] .
\end{aligned}
$$

The counterpart of Theorem 3 for the case of LPTV precoding then follows immediately from the results of [2] [3].

Theorem 3: Let $x[n]$ be a sequence of zero-mean uncorrelated symbols, each with energy $\mathcal{E}_{s}$. Furthermore, for every $M$, let $a_{m}$ for $m=0,1, \ldots, M-1$ be a collection of independent zero-mean complex-valued Gaussian random variables with variance $\sigma_{a}^{2}$. Finally, suppose $\tilde{b}[n]$ is an admissible equalizer, that the length- $M$ antenna precoder signature sequences form an orthonormal set, and that maximally spread prefilters of spread $M$ are used. Then, as $M \rightarrow \infty$, we have that $\hat{x}[n]$ defined via (44) with (47), (28), and (32) satisfies, for each $n$,

$$
\hat{x}[n] \stackrel{\text { m.s. }}{\longrightarrow} \mu_{c} x[n]+v[n]
$$

where $v[n]$ is a complex-valued, marginally Gaussian, zeromean white noise sequence, uncorrelated with the input symbol stream $x[n]$, and having variance

$$
\operatorname{var} v[n]=\mathcal{E}_{s} \sigma_{c}^{2}+\mathcal{N}_{0}\left(\sigma_{b}^{2}+\left|\mu_{b}\right|^{2}\right) .
$$

The SNR in the equivalent channel of Theorem 3 then follows analogously as

$$
\begin{aligned}
\gamma(b) & =\frac{\left|\mu_{c}\right|^{2}}{\sigma_{c}^{2}+\xi_{0}\left(\sigma_{b}^{2}+\left|\mu_{b}\right|^{2}\right)} \\
& =\frac{|E[a[n] b[n]]|^{2}}{\operatorname{var}[a[n] b[n]]+\xi_{0} E\left[|b[n]|^{2}\right]}
\end{aligned}
$$

where, again,

$$
\xi_{0}=\mathcal{N}_{0} / \mathcal{E}_{s}
$$

and is maximum when [2]

$$
\tilde{b}[n] \propto \frac{\tilde{a}^{*}[n]}{|\tilde{a}[n]|^{2}+\xi_{0}} .
$$

Theorem 3 establishes that the generalized transmit diversity schemes we have developed in this section have the same attractive asymptotic characteristics as those considered in Section IV. However, for finite transmit antenna arrays, these generalized schemes have some important advantages. In particular, unlike the optimized transmit diversity schemes based on LTI precoders, those we have optimized from an LPTV framework have the characteristic that the system delay is finite. Indeed, the fading equalizer (52) introduces no delay, whereas the pre- and post-filtering each introduce delay $M$; therefore, the overall delay is proportional to the number of antennas.

\section{Performance}

The infinite-diversity results described in Section IV-B provide useful bounds on what can be achieved using finite transmit antenna diversity. In this section, we present empirical results on the finite-diversity performance when the optimized LPTV antenna precoders are used. In particular, using maximally spread prefilters (with $K=2$ ) and the optimum equalizers, the performance of LPTV antenna precoding is depicted in Fig. 7 for several different antenna array sizes. In addition, the performance without antenna diversity $(M=1)$ and the performance with infinite transmit diversity $(M \rightarrow \infty)$ is superimposed. Note that while additional antennas invariably give better performance, Fig. 7 indicates that there are diminishing returns beyond a moderate value of $M$. Moreover, hardware costs and system delay constraints typically limit values of $M$ that can be used in practice.

For comparison, Fig. 8 shows the corresponding (normalized) performance of systems employing traditional receive antenna diversity with maximal ratio combining for various antenna array sizes. Comparisons of Figs. 7 and 8, particularly when power asymmetries are taken into account, suggest that, when available, receive diversity is more effectively exploited via linear processing. However, as our results also demonstrate, in many applications such as broadcasting where receive diversity is less practical, exploiting transmit diversity 


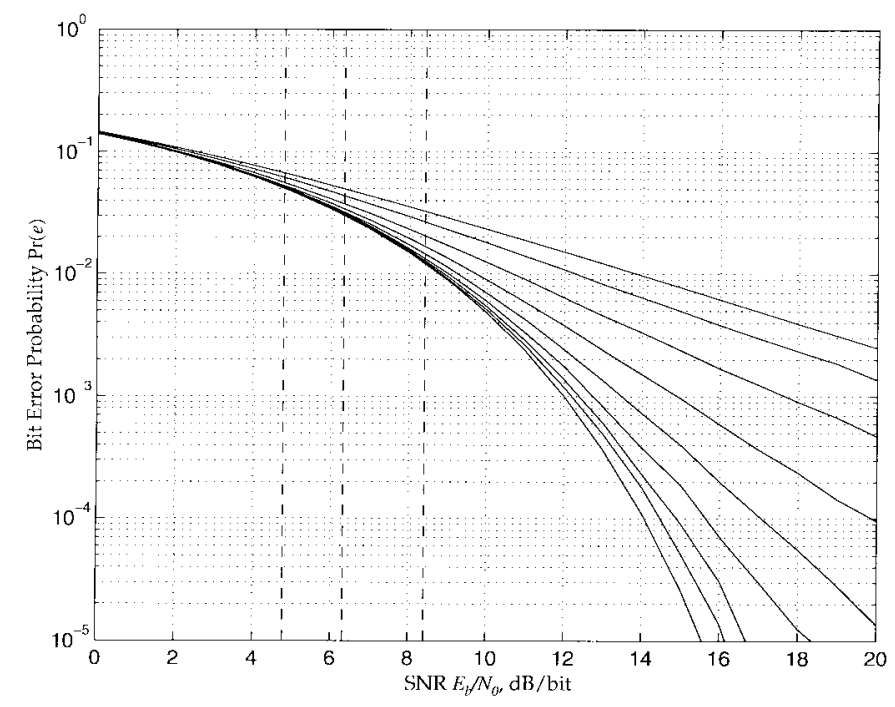

Fig. 7. Bit error probabilities using uncoded QPSK on the Rayleigh fading channel with transmit diversity in the form of linear antenna precoding with linear equalization. The top curve corresponds to the performance without antenna diversity $(M=1)$, while the bottom curve indicates the performance bound corresponding to an infinite transmit diversity $(M \rightarrow \infty)$. The successively lower curves between these two extremes represent the performance obtained using $M=2,4,8,16,32,64$, and 128, transmit antennas, respectively. From left to right, the dashed vertical lines denote the capacities $\mathcal{C}_{\mathrm{T}}, \mathcal{C}_{\mathrm{L}}$, and $\mathcal{C}$, respectively.

using even only a few antennas can provide a dramatic reduction in transmit power requirements for a given bit error rate and at a very modest cost in terms of additional system hardware. More generally, our results suggest that for a number of applications, the best cost-performance tradeoff may be achieved by simultaneously exploiting both forms of spatial diversity.

\section{CONCLUding REMARKS}

This paper has developed computationally efficient transmit diversity strategies that can be used to substantially mitigate the effects of fading in wireless communications applications. Moreover, these techniques naturally lend themselves to practical and efficient DSP-based implementations.

Several issues remain to be investigated and represent important directions for further research. One important example is the issue of channel measurement and tracking. In practice, the channel fading coefficients required by the receiver must be estimated from the received data, either using training data or blind. Future work must explore suitable adaptive equalizer structures based on, for example, LMS or RLS algorithms and assess the impact of these structures on overall system performance and the associated sensitivity issues described earlier.

In addition, the work here has focussed on nonselective fading channels, which is appropriate in low-bandwidth wireless applications, where the transmitter and receiver are stationary. In wideband scenarios, the channel becomes frequency selective, whereas in mobile applications, the channel becomes time selective. In principle, the techniques developed in this paper can be extended to accommodate this broader class of chan-

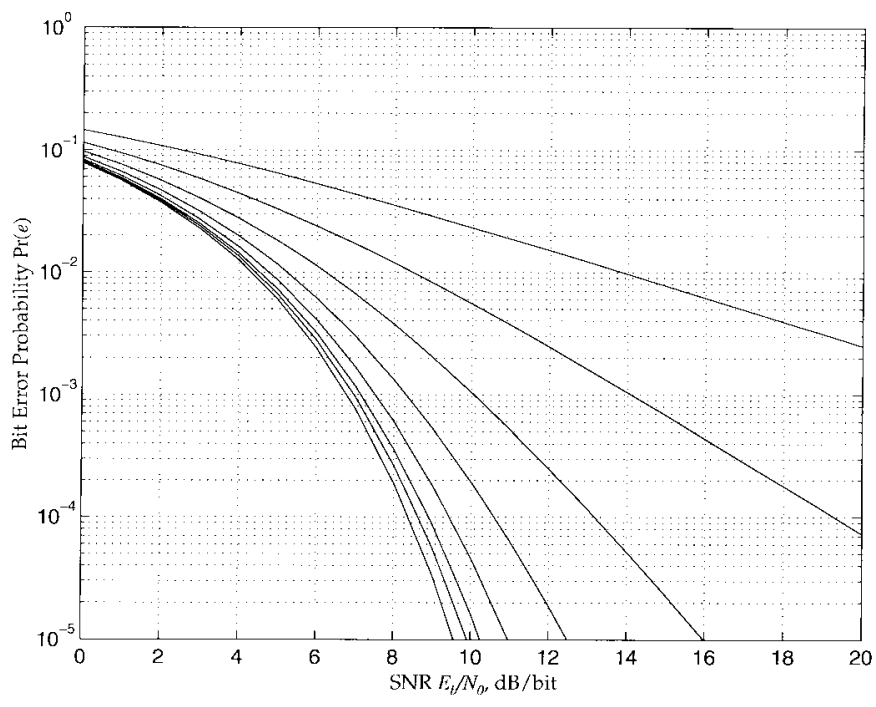

Fig. 8. Bit error probabilities using uncoded QPSK on the Rayleigh fading channel with (normalized) receive diversity using maximal ratio combining. The top curve corresponds to the performance without antenna diversity $(M=1)$, while the bottom curve indicates the performance bound corresponding to an infinite receive diversity $(M \rightarrow \infty)$. The successively lower curves between these two extremes represent the performance obtained using $M=2,4,8,16,32,64$, and 128 , receive antennas, respectively.

nels. Developing and evaluating these extensions represents another rich direction for further research.

As a final example, the transmit diversity schemes in this work introduce a delay proportional to the number of antennas but do not expand bandwidth. In principle, it is possible to trade off delay for bandwidth expansion when desirable. As an example, a transmit diversity scheme described by Jakes [4], whereby the symbol stream is transmitted in distinct frequency bands at the different antennas, incurs no additional delay but requires a bandwidth expansion proportional to the number of antennas. However, schemes of this type are rather inefficient in their use of the additional bandwidth and are therefore inherently suboptimum. Exploring how the transmit diversity schemes developed in this paper can be generalized to allow for efficient delay-bandwidth tradeoffs, and more than compensate for the increase receiver noise power due to bandwidth expansion, is another promising direction for further research.

\section{APPENDIX A PROOF OF THEOREM 1}

We verify that the conditions (4) are satisfied when the signature sequences are orthogonal to one another (and normalized), i.e.,

$$
\sum_{n=0}^{M-1} h_{m}[n] h_{k}^{*}[n]=\delta[m-k] .
$$

In this case, the rows of $\mathbf{H}$ are orthogonal; therefore, $\mathbf{H}$ is an unitary matrix. Then, using (3), we obtain

$$
\rho_{M}(\omega, \nu)=\sum_{n=0}^{M-1} \sum_{l=0}^{M-1} e^{-j(\omega n-\nu l)} \phi_{M}[n, l]
$$


where

$$
\phi_{M}[n, l]=\sum_{m=0}^{M-1} h_{m}[n] h_{m}^{*}[l]
$$

However, since $\mathbf{H}$ is a unitary matrix, its columns are also orthogonal, and hence, $\phi_{M}[n, l]=\delta[n-l]$. Thus, (53) simplifies to

$$
\left|\rho_{M}(\omega, \nu)\right|=\left\{\left|\frac{\sin [(\omega-\nu) M / 2]}{M \sin [(\omega-\nu) / 2]}\right| \begin{array}{ll}
\omega=\nu \\
\omega \neq \nu
\end{array}\right.
$$

which, as is readily verified, satisfies (4).

\section{APPENDIX B}

\section{PROOF OF THEOREM 2}

We begin with a pair of lemmas that will be useful in the sequel.

Lemma 1: Let $A(\omega)$ be a complex-valued $2 \pi$-periodic zeromean Gaussian random process with variance $\sigma_{a}^{2}$ and normalized correlation function denoted by

$$
\rho(\omega, \nu)=\frac{E\left[A(\omega) A^{*}(\nu)\right]}{\sigma_{a}^{2}} .
$$

Furthermore, let a new random process $\tilde{D}(\omega)$ be defined via

$$
\tilde{D}(\omega)=g(A(\omega))
$$

for some function $g(\cdot)$ such that

$$
\begin{gathered}
E[\tilde{D}(\omega)]=0 \\
E[|\tilde{D}(\omega)|]<\infty \\
\operatorname{var} \tilde{D}(\omega)<\infty .
\end{gathered}
$$

Then, if ${ }^{12} \rho(\omega, \nu) \stackrel{\text { p.w.a.e. }}{\longrightarrow} 0$, we have that

$$
\mathcal{I}=\frac{1}{4 \pi^{2}} \int_{-\pi}^{\pi} \int_{-\pi}^{\pi}\left|E\left[\tilde{D}(\omega) \tilde{D}^{*}(\nu)\right]\right| d \omega d \nu \rightarrow 0 .
$$

Proof: First, we note that

$$
\left|E\left[\tilde{D}(\omega) \tilde{D}^{*}(\nu)\right]\right| \leq \operatorname{var} \tilde{D}(\omega)<\infty
$$

where the last inequality follows from (59). In turn, using (61) with the bounded convergence theorem [15], we obtain

$$
\mathcal{I} \rightarrow 0
$$

provided

$$
E\left[\tilde{D}(\omega) \tilde{D}^{*}(\nu)\right] \stackrel{\text { p.w.a.e. }}{\longrightarrow} 0 .
$$

Hence, it suffices to show that $\rho(\omega, \nu) \stackrel{\text { p.w.a.e. }}{\longrightarrow} 0$ implies (62). Letting $x=A(\omega)$ and $y=A(\nu)$, where $\omega \neq \nu$, we see that it is sufficient to show that

$$
\begin{aligned}
& \lambda(\rho) \triangleq E\left[\tilde{D}(\omega) \tilde{D}^{*}(\nu)\right]=\int_{-\infty}^{+\infty} \int_{-\infty}^{+\infty} g(x) g^{*}(y) \\
& \frac{1}{\left(2 \pi \sigma_{a}^{2}\right)^{2}\left(1-|\rho|^{2}\right)} \exp \left[-\frac{|x|^{2}+|y|^{2}-2 \operatorname{Re}\left\{\rho x^{*} y\right\}}{2 \sigma_{a}^{2}\left(1-|\rho|^{2}\right)}\right] d x d y
\end{aligned}
$$

\footnotetext{
${ }^{12} \mathrm{We}$ use $\stackrel{\text { p.w.a.e. }}{\longrightarrow}$ to denote pointwise convergence almost everywhere.
}

satisfies $\lim _{\rho \rightarrow 0} \lambda(\rho)=0$.

Now, we exploit the inequality

$$
\frac{|x|^{2}+|y|^{2}}{1+|\rho|} \leq \frac{|x|^{2}+|y|^{2}-2 \operatorname{Re}\left\{\rho x^{*} y\right\}}{1-|\rho|^{2}}
$$

which is verified as follows:

$$
\begin{aligned}
& \left(|x|^{2}+|y|^{2}\right)\left[\frac{1}{1-|\rho|^{2}}-\frac{1}{1+|\rho|}\right]-\frac{2 \operatorname{Re}\left\{\rho x^{*} y\right\}}{1-|\rho|^{2}} \\
& =\frac{\left(|x|^{2}+|y|^{2}\right)|\rho|-2 \operatorname{Re}\left\{\rho x^{*} y\right\}}{1-|\rho|^{2}} \\
& =\frac{|\rho|}{1-|\rho|^{2}}\left[|x|^{2}+|y|^{2}-2 \operatorname{Re}\left\{e^{j \angle \rho} x^{*} y\right\}\right] \\
& =\frac{|\rho|}{1-|\rho|^{2}}\left|x-e^{j \angle \rho} y\right|^{2} \geq 0
\end{aligned}
$$

where $\rho=|\rho| e^{j L \rho}$. Using, in order, the triangle inequality and (64), we obtain

$$
|\lambda(\rho)| \leq \lambda^{\prime}(\rho)
$$

where

$$
\begin{aligned}
& \lambda^{\prime}(\rho)=\frac{1}{1-|\rho|} \int_{-\infty}^{+\infty} \int_{-\infty}^{+\infty}|g(x)|\left|g^{*}(y)\right| \\
& \frac{1}{\left(2 \pi \sigma_{a}^{2}\right)^{2}(1+|\rho|)} \exp \left[-\frac{|x|^{2}+|y|^{2}}{2 \sigma_{a}^{2}(1+|\rho|)}\right] d x d y .
\end{aligned}
$$

However, $\lambda^{\prime}(\rho)$ is a continuous function of $\rho$ on $0 \leq|\rho|<1$, and

$$
\lambda^{\prime}(0)=(E[|g(x)|])^{2}<\infty
$$

where the right inequality in (67) follows from (58). Hence, we conclude that $\lambda^{\prime}(\rho)$ is upper bounded in a neighborhood of $\rho=0$. This result allows us to conclude from the dominated convergence theorem [15] that we can evaluate $\lambda(\rho)$ as $\rho \rightarrow \infty$ by bringing the limit inside the integral in (63), yielding

$$
\lim _{\rho \rightarrow 0} \lambda(\rho)=\left[\int_{-\infty}^{+\infty} g(z) \frac{1}{2 \pi \sigma_{a}^{2}} e^{-|z|^{2} /\left(2 \sigma_{a}^{2}\right)} d z\right]^{2}=0
$$

where the last equality follows from (57).

Lemma 2: If $B(\omega)$ is an admissible equalizer in the sense of Definition 2, then the admissibility conditions on $f(\cdot)$ imply that the zero-mean random processes $\tilde{C}(\omega)$ and

$$
\begin{aligned}
& \tilde{D}_{1}(\omega)=|B(\omega)|^{2}-E\left[|B(\omega)|^{2}\right] \\
& \tilde{D}_{2}(\omega)=|\tilde{C}(\omega)|^{2}-\sigma_{c}^{2}
\end{aligned}
$$

with

$$
\widetilde{C}(\omega)=C(\omega)-\mu_{c}
$$

satisfy

$$
\begin{array}{cc}
\operatorname{var} \tilde{C}(\omega)<\infty & E[|\tilde{C}(\omega)|]<\infty \\
\operatorname{var} \tilde{D}_{1}(\omega)<\infty & E\left[\left|\tilde{D}_{1}(\omega)\right|\right]<\infty \\
\operatorname{var} \tilde{D}_{2}(\omega)<\infty & E\left[\left|\tilde{D}_{2}(\omega)\right|\right]<\infty
\end{array}
$$


Proof: The proof is straightforward and follows from observing that

$$
\begin{aligned}
\tilde{C}(\omega) & =g_{0}(A(\omega)) \\
\tilde{D}_{1}(\omega) & =g_{1}(A(\omega)) \\
\tilde{D}_{2}(\omega) & =g_{2}(A(\omega))
\end{aligned}
$$

where

$$
\begin{aligned}
& g_{0}(z)=z f(z)-\kappa_{0} \\
& g_{1}(z)=|f(z)|^{2}-\kappa_{1} \\
& g_{2}(z)=\left|z f(z)-\mu_{c}\right|^{2}-\kappa_{2}
\end{aligned}
$$

and where the $\kappa_{i}$ are finite constants. From (74), we see that the $g_{i}(\cdot)$ satisfy the admissibility conditions of Definition 2 whenever $f(\cdot)$ does. Using these conditions in the expressions

$$
\begin{aligned}
\operatorname{var} \tilde{C}(\omega) & =\frac{1}{2 \pi \sigma_{a}^{2}} \int_{-\infty}^{+\infty} g_{0}^{2}(z) e^{-|z|^{2} /\left(2 \sigma_{a}^{2}\right)} d z \\
E[|\tilde{C}(\omega)|] & =\frac{1}{2 \pi \sigma_{a}^{2}} \int_{-\infty}^{+\infty}\left|g_{0}(z)\right| e^{-|z|^{2} /\left(2 \sigma_{a}^{2}\right)} d z
\end{aligned}
$$

and

$$
\begin{aligned}
\operatorname{var} \tilde{D}_{i}(\omega) & =\frac{1}{2 \pi \sigma_{a}^{2}} \int_{-\infty}^{+\infty} g_{i}^{2}(z) e^{-|z|^{2} /\left(2 \sigma_{a}^{2}\right)} d z \\
E\left[\left|\tilde{D}_{i}(\omega)\right|\right] & =\frac{1}{2 \pi \sigma_{a}^{2}} \int_{-\infty}^{+\infty}\left|g_{i}(z)\right| e^{-|z|^{2} /\left(2 \sigma_{a}^{2}\right)} d z
\end{aligned}
$$

for $i=1,2$, we obtain our desired results.

We now proceed to a proof of our main result.

First, we write

$$
\hat{x}[n]=v_{1}[n]+z[n]
$$

where

$$
v_{1}[n]=b[n] * w[n]
$$

and

$$
z[n]=c[n] * x[n]
$$

with

$$
c[n]=b[n] * a[n] .
$$

Let us consider $v_{1}[n]$ as defined in (76) first.

First, we obtain the mean and covariance, respectively, of $v_{1}[n]$ given an effective channel response $a[\cdot]$ as

$$
E\left[v_{1}[n] \mid a\right]=0
$$

and

$$
R_{v_{1} v_{1} \mid a}[n]=\frac{\mathcal{N}_{0}}{2 \pi} \int_{-\pi}^{\pi}|B(\omega)|^{2} e^{j \omega n} d \omega .
$$

In turn, averaging over the possible channel responses $a[\cdot]$, we obtain

$$
\begin{aligned}
& E\left[R_{v_{1} v_{1} \mid a}[n]\right]=E\left[|B(\omega)|^{2}\right] \frac{\mathcal{N}_{0}}{2 \pi} \int_{-\pi}^{\pi} e^{j \omega n} d \omega \\
& =\mathcal{N}_{0} E\left[|B(\omega)|^{2}\right] \delta[n]
\end{aligned}
$$

where we have used the fact that since the mean and variance of $A(\omega)$ are independent of $\omega$, so are those of $B(\omega)$. Next, we obtain

$$
\begin{aligned}
\tilde{R}_{v_{1} v_{1} \mid a}[n] & \triangleq R_{v_{1} v_{1} \mid a}[n]-E\left[R_{v_{1} v_{1} \mid a}[n]\right] \\
& =\frac{\mathcal{N}_{0}}{2 \pi} \int_{-\pi}^{\pi} \tilde{D}_{1}(\omega) e^{j \omega n} d \omega
\end{aligned}
$$

where $\tilde{D}_{1}(\omega)$ is as defined in (68a). Then,

$$
\begin{aligned}
& \operatorname{var} R_{v_{1} v_{1} \mid a}[n]=E\left[\left|\tilde{R}_{v_{1} v_{1} \mid a}\right|^{2}\right] \\
& =\left(\frac{\mathcal{N}_{0}}{2 \pi}\right)^{2} \int_{-\pi}^{\pi} \int_{-\pi}^{\pi} E\left[\tilde{D}_{1}(\omega) \tilde{D}_{1}(\nu)\right] e^{j(\omega-\nu) n} d \omega d \nu
\end{aligned}
$$

Using (4) and applying, in turn, Lemmas 2 and 1 to (82), we then obtain, for each $n$

$$
\operatorname{var} R_{v_{1} v_{1} \mid a}[n] \rightarrow 0
$$

Hence, combining (81) with (83), we have, for any particular channel response $a[\cdot]$,

$$
\begin{aligned}
& R_{v_{1} v_{1} \mid a}[n] \stackrel{\mathrm{m} . s}{\longrightarrow} \mathcal{N}_{0} E\left[|B(\omega)|^{2}\right] \delta[n] \\
& \quad=\mathcal{N}_{0}\left(\sigma_{b}^{2}+\left|\mu_{b}\right|^{2}\right) \delta[n]
\end{aligned}
$$

for each $n$.

Looking next at $z[n]$ as defined in (77), we express $z[n]$ in the form

$$
z[n]=v_{2}[n]+\mu_{c} x[n]
$$

Then

$$
v_{2}[n]=\tilde{c}[n] * x[n]
$$

where

$$
\tilde{c}[n]=c[n]-\mu_{c} \delta[n]=c[n]-E[c[n]]
$$

Therefore, $\widetilde{c}[n]$ is a zero-mean sequence.

Again, for a fixed realization of $a[\cdot]$ (and hence $c[\cdot]$ ), we have

$$
E\left[v_{2}[n] \mid a\right]=\tilde{c}[n] * E[x[n]]=0
$$

and

$$
R_{v_{2} v_{2} \mid a}[n]=\frac{\mathcal{E}_{s}}{2 \pi} \int_{-\pi}^{\pi}|\tilde{C}(\omega)|^{2} e^{j \omega n} d \omega
$$


However, (88) is asymptotically independent of $a[\cdot]$. To see this, first note that

$$
E\left[R_{v_{2} v_{2} \mid a}[n]\right]=\frac{\mathcal{E}_{s}}{2 \pi} \int_{-\pi}^{\pi} \sigma_{c}^{2} e^{j \omega n} d \omega=\mathcal{E}_{s} \sigma_{c}^{2} \delta[n] .
$$

Then, since

$$
\begin{aligned}
\tilde{R}_{v_{2} v_{2} \mid a}[n] & \triangleq R_{v_{2} v_{2} \mid a}[n]-E\left[R_{v_{2} v_{2} \mid a}[n]\right] \\
& =\frac{\mathcal{E}_{s}}{2 \pi} \int_{-\pi}^{\pi} \tilde{D}_{2}(\omega) e^{j \omega n} d \omega
\end{aligned}
$$

where $\tilde{D}_{2}(\omega)$ is as defined in $(68 \mathrm{~b})$, we have

$$
\begin{aligned}
& \operatorname{var} R_{v_{2} v_{2} \mid a}[n]=E\left[\left|\tilde{R}_{v_{1} v_{1} \mid a}\right|^{2}\right] \\
& \quad=\left(\frac{\mathcal{E}_{s}}{2 \pi}\right)^{2} \int_{-\pi}^{\pi} \int_{-\pi}^{\pi} E\left[\tilde{D}_{2}(\omega) \tilde{D}_{2}(\nu)\right] e^{j(\omega-\nu) n} d \omega d \nu
\end{aligned}
$$

Hence, again using (4) and applying, in turn, Lemmas 2 and 1 to (90), we then obtain, for each $n$,

$$
\operatorname{var} R_{v_{2} v_{2} \mid a}[n] \rightarrow 0
$$

Hence, combining (89) with (91), we have, for any particular channel response $a[\cdot]$,

$$
R_{v_{2} v_{2} \mid a}[n] \stackrel{\text { m.s. }}{\longrightarrow} \mathcal{E}_{s} \sigma_{c}^{2} \delta[n]
$$

for each $n$.

Thus, we can write

$$
\hat{x}[n]=\mu_{c} x[n]+v[n]
$$

where

$$
v[n]=v_{1}[n]+v_{2}[n]
$$

is the equivalent noise. However, since $x[n]$ and $w[n]$ are statistically independent,

$$
E\left[v_{1}[n] v_{2}^{*}[k] \mid a\right]=0, \quad \text { for all } n \text { and } k
$$

and hence

$$
R_{v v \mid a}[n]=R_{v_{1} v_{2} \mid a}[n]+R_{v_{2} v_{2} \mid a}[n]
$$

which, using (84) and (92), yields (13).

Finally, we need to show that for a given realization of the channel response $a[n]$ that $x[n]$ and $v[n]$ are asymptotically uncorrelated. Due to (94), it suffices to show that $x[n]$ is asymptotically uncorrelated with $v_{1}[n]$ and $v_{2}[n]$ individually.

First, using (76), we have

$$
E\left[v_{1}[n] x^{*}[k]\right]=\sum_{l} E\left[w[l] x^{*}[k]\right] b[n-l]=0
$$

where the last equality follows from the fact that the processes $w[n]$ and $x[n]$ are statistically independent.
Next, using (86), we have

$$
E\left[v_{2}[n] x^{*}[k]\right]=\sum_{l} E\left[x[l] x^{*}[k]\right] \tilde{c}[n-l]=\mathcal{E}_{s} \tilde{c}[n-k]
$$

where the last equality follows from the fact that the symbol stream $x[n]$ is white. Thus, it remains only to show that $\tilde{c}[n] \stackrel{\text { m.s. }}{\longrightarrow} 0$ for all $n$.

To see this, we first note that

$$
E[\tilde{c}[n]]=0 .
$$

Next,

$$
\operatorname{var} \tilde{c}[n]=\left(\frac{1}{2 \pi}\right)^{2} \int_{-\pi}^{\pi} \int_{-\pi}^{\pi} E\left[\tilde{C}(\omega) \tilde{C}^{*}(\nu)\right] e^{j(\omega-\nu) n} d \omega d \nu .
$$

Hence, again using (4) and applying, in turn, Lemmas 2 and 1 to (96), we then obtain, for each $n$

$$
\operatorname{var} \widetilde{c}[n] \rightarrow 0
$$

Hence, combining (95) with (97), we have

$$
\tilde{c}[n] \stackrel{\text { m.s. }}{\longrightarrow} 0
$$

for each $n$.

\section{REFERENCES}

[1] E. Biglieri, D. Divsalar, P. McLane, and M. Simon, Introduction to Trellis-Coded Modulation with Applications. New York: MaxwellMacmillan, 1991.

[2] G. W. Wornell, "Spread-response precoding for communication over fading channels," IEEE Trans. Inform. Theory, vol. 42, pp. 488-501, Mar. 1996.

[3] _ _ "Spread-signature CDMA: Efficient multiuser communication in the presence of fading," IEEE Trans. Inform. Theory, vol. 41, pp. 1418-1438, Sept. 1995.

[4] W. C. Jakes, Ed., Microwave Mobile Communications. New York: Wiley, 1974

[5] D. Gerlach and A. Paulraj, "Adaptive transmitting antenna methods for multipath environments," in Proc. IEEE GLOBECOM, 1994.

[6] A. Wittneben, "Basestation modulation diversity for digital simulcast," in Proc. Vehic. Technol. Soc. Conf., 1991, pp. 848-853.

[7] J. H. Winters, "The diversity gain of transmit diversity in wireless systems with Rayleigh fading," in Proc. Int. Conf. Commun., 1994, pp. $1121-1125$.

[8] A. Hiroike, F. Adachi, and N. Nakajima, "Combined effects of phase sweeping tranmitter diversity and channel coding," IEEE Trans. Vehic. Technol., vol. 41, pp. 170-176, May 1992.

[9] V. Weerackody, "Diversity for the direct-sequence spread spectrum system using multiple tranmit antennas," in Proc. Int. Conf. Commun., 1993, pp. 1775-1779.

[10] A. Narula, M. D. Trott, and G. W. Wornell, "Information-theoretic analysis of multiple-antenna transmission divesity for fading channels," in Proc. Int. Symp. Inform. Theory Appl., Sept. 1996.

[11] A. Narula, M. D. Trott, and G. W. Wornell, "Information-theoretic analysis of multiple-antenna transmission diversity," submitted to IEEE Trans. Inform. Theory.

[12] E. A. Lee and D. G. Messerschmitt, Digital Communication. Boston, MA: Kluwer, 1994, 2nd ed.

[13] J. G. Proakis, Digital Communications. New York: McGraw-Hill, 1989, 2nd ed.

[14] M. Abramowitz and I. A. Stegun, Eds., Handbook of Mathematical Functions. New York: Dover, 1965.

[15] D. C. Champeney, A Handbook of Fourier Theorems. Cambridge, U.K.: Cambridge Univ. Press, 1987. 


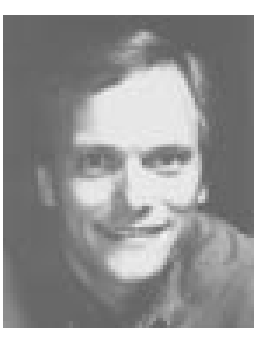

Gregory W. Wornell (M'91) was born in Montréal, P.Q., Canada, in 1962. He received the B.A.Sc. degree (with honors) from the University of British Columbia, Vancouver, B.C., Canada, and the S.M. and Ph.D. degrees from the Massachusetts Institute of Technology (MIT), Cambridge, all in electrical engineering, in 1985, 1987, and 1991, respectively.

Since 1991, he has been with the faculty of the Department of Electrical Engineering and Computer Science at MIT, where he is currently Cecil and Ida Green Career Development Associate Professor. During the 1992-1993 academic year, he was on leave at AT\&T Bell Laboratories, Murray Hill, NJ, and during 1990, he was a Visiting Investigator at the Woods Hole Oceanographic Institute, Woods Hole, MA. His current research interests include signal processing, wireless and braodband communications, and applications of fractal geometry and nonlinear dynamical system theory in these areas. He is the author of the monograph Signal Processing with Fractals: A Wavelet-Based Approach (Englewood Cliffs, NJ: Prentice-Hall). $\mathrm{He}$ is also a consultant to industry and holds one patent in the area of communications. Three other patents are pending.

Dr. Wornell has received many awards for teaching and research including the MIT Goodwin Medal for "conspicuously effective teaching" (1991), the ITT Career Development Chair at MIT (1993), an NSF Faculty Early Career Development Award (1995), an ONR Young Investigator Award (1996), and the MIT Junior Bose Award for Excellence in Teaching (1996). He is a member of Tau Beta Pi and Sigma Xi.

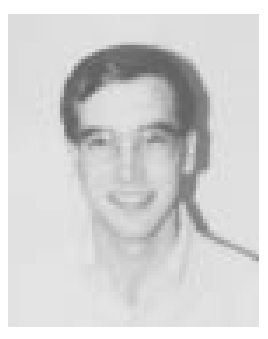

Mitchell D. Trott (M'93) received the B.S. and M.S. degrees in systems engineering from Case Western Reserve University, Cleveland, $\mathrm{OH}$, in 1987 and 1988, respectively, and the Ph.D. degree in electrical engineering from Stanford University, Stanford, CA, in 1992.

$\mathrm{He}$ is currently an Assistant Professor in the Department of Electrical Engineering and Computer Science, Massachusetts Institute of Technology (MIT), Cambridge. His research interests include coding theory, algebraic system theory, and mobile

radio communications.

Dr. Trott received an NSF Young Investigator Award in 1994. 